\title{
Acoustic streaming in a waveguide with slowly varying height
}

\author{
Charles Thompson \\ Department of Engineering Science and Mechanics, Virginia Polytechnic Institute and State University, \\ Blacksburg, Virginia 24061
}

(Received 24 February 1983; accepted for publication 21 June 1983)

\begin{abstract}
An analysis of acoustic streaming in a two-dimensional waveguide having slowly varying height is presented. Special attention is paid to waveguides with cross sections that are small compared to the acoustic and/or wall wavelengths. It is shown that the dynamic behavior of the enclosed fluid can be parameterized by the values of three small parameters, $\epsilon, 1 / S$, and $1 / R$, where $\epsilon$ is the ratio of the typical duct height $H_{0}$ to the wall wavelength $L_{0}, 1 / S$ is the ratio of the typical oscillatory particle displacement $U_{0} / \omega$ to the typical duct height $H_{0}$ and $1 / R$ is the ratio of the oscillatory boundary layer thickness $l_{v}$ to the typical duct height $H_{0}$. An analytical solution describing the streaming flow in the duct is given in terms of a regular perturbation sequence in $\epsilon$. It is shown that the oscillatory pressure must satisfy the lossy Webster horn equation to $O\left(\epsilon^{2}\right)$ if the no slip boundary condition is to be satisfied. Outside the boundary layer it is shown that the time averaged slip velocity is the sum of two terms. The first term is proportional to the product of the incident and reflected wave amplitudes. The second term is proportional to the difference between the incident and reflected acoustic intensity of the wave. For small values of $1 / S, 1 / R$, and $\epsilon$ the streaming solution given is shown to be valid until $\epsilon R / S^{2}$ becomes of $O(1)$.
\end{abstract}

PACS numbers: $43.25 . \mathrm{Nm}$

\section{INTRODUCTION}

Acoustic streaming caused by a harmonically excited standing wave in a uniform duct was first analyzed by Rayleigh ${ }^{1}$ in 1883 . More recently, there has been increased interest in the role acoustic streaming developed in a purely oscillatory flow field, plays in problems dealing with biophysical fluid transport. Haselton and Schuer $^{2}$ pointed out that acoustic streaming in a bronchial tube with a bifurcation, aids in gas exchange with the environment and is the major cause of inhaled aerosol motion. In blood flow, Caro, Fitzgerald, and Schroter ${ }^{3}$ discussed the influence the wall sheer stress has on the development of atherosclerosis. However, these investigators based their conclusion on experiments made under steady flow conditions. Actually, if the distensibility of the vessel is taken into account, the oscillatory flow and the acoustic streaming it generates may contribute to the wall sheer stress. However, in many problems involving biophysical mass transport, the nonuniformity of the tube shape causes Rayleigh's solution to become inapplicable.

There has also been some discussion by Wang ${ }^{4}$ in 1968 and Secomb ${ }^{5}$ in 1978 on the maximum amplitude for which Rayleigh's solution is valid. Wang noted that Rayleigh's result should be valid only up to $R / S^{2}=O(1)$, where $R$ is the acoustic Reynolds number and $S$ is the Strouhal number. Secomb on the other hand stated that Rayleigh's result is valid to $R / S^{2}=O(\infty)$ if the acoustic wavelength is large compared with respect to the height of the tube. However, neither of these amplitude bounds is completely correct for the problem we will address here. In obtaining each of these amplitude bounds, both investigations omitted two important parameters, namely, the acoustic wavelength and the wall slope.

In this paper, we will be concerned with the steady flow induced by the harmonic excitation of a fluid in a rigid two- dimensional waveguide (see Fig. 1). The effect of viscosity on the oscillatory flow is assumed to be confined to a thin layer at the walls. Therefore, the Reynolds number $R$, is assumed to have a value much larger than one. The thickness of the Stokes layer will be of order $1 / \sqrt{R}$. We will develop the streaming solution for wall slope and the ratio of height of the duct to acoustic wavelength being of order $\epsilon$. A regular perturbation solution in $\epsilon$ for the streaming in a waveguide with slowly varying height is presented.

Schlichting, ${ }^{6}$ Riley, ${ }^{7}$ and Stuart ${ }^{8}$ have shown that acoustic streaming around a cylinder is dependent on the finite magnitude of $U_{0} /(\omega d)$ where $U_{0} / \omega$ is the amplitude of the oscillatory fluid displacement and $d$ is the cylinder diameter. For an acoustic wave in a waveguide with slowly varying height the appropriate replacement for the diameter $d$ is the wall's maximum radius of curvature $r_{c}$ which is proportional to $L_{0}^{2} / H_{0}$. Thus $\epsilon^{2} / S$ represents the appropriate amplitude parameter. For $S$ fixed, acoustic streaming vanishes as $\epsilon$ approaches zero. Hence, one expects acoustic streaming to appear in higher order terms in $\epsilon$.

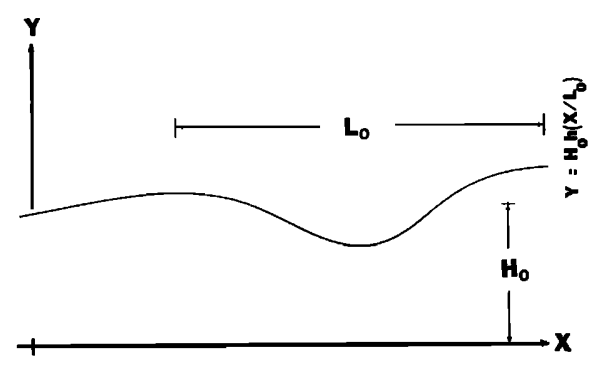

FIG. 1. Schematic of a waveguide having a slowly varying height. The height if the guide is given by the function $Y=H_{0} h\left(X / L_{0}\right)$. 
In Sec. I we will make a brief statement of the equations of motion in dimensional form. We will also discuss the issues involved in sound generated flow. It will be shown that acoustic streaming is driven by the gradient of the Reynolds stress, $\overline{\rho V_{i} V_{j}}$. Section II will be devoted to developing the nondimensional equations which govern fluid motion in a two-dimensional waveguide, in the long wavelength limit. Upon nondimensionalization we will show that in a waveguide the fluid's behavior is parameterized by the numerical value of three dimensionless parameters $S, R$, and $\epsilon$ where $S$ is the Strouhal number, $R$ the oscillatory Reynold's number, and $\epsilon$ is the ratio of the duct height to wall wavelength.

We will assume that both unsteady and steady variables can be represented by truncated geometric sequences in $\epsilon$. These sequences are

$$
(u, v, p, \bar{u}, \bar{v}, \bar{p}) \sim E_{3}(u, v, p, \bar{u}, \bar{v}, \bar{p}),
$$

where

$$
E_{N} f\left(\mathbf{x}, \frac{1}{R}, \frac{1}{S} ; \epsilon\right)=\sum_{n=0}^{N} \epsilon^{n} f_{n}\left(\mathbf{x}, \frac{1}{R}, \frac{1}{S}\right)+O\left(\epsilon^{N+1}\right) \text {. }
$$

By substituting these sequences into the nondimensional equations given in Sec. II and equating like terms in $\epsilon$, we obtain a set of partial differential equations governing the values of the coefficients. In Secs. III-VI we will present the solution for the coefficients of the aforementioned asymptotic sequence.

In Sec. VII we will parameterize the region of validity of our perturbation solution. We will show that our solution under the restriction of large $R$ and $S$ and small $\epsilon$ is valid to $R / S^{2}=O(1 / \epsilon)$. Hence, the amplitude bounds obtained by Wang $^{4}$ and Secomb ${ }^{5}$ represent the limiting value of the streaming Reynolds number as $\epsilon$ tends to one and zero, respectively.

\section{STATEMENT OF THE EQUATIONS OF MOTION}

In this section we will state the equations of motion for a viscous fluid. Simplification of the exact equations will be made. The conditions under which the approximate equations are valid will be denoted explicitly. form is

The statement of conservation of mass in Reynolds

$$
\frac{\partial \rho}{\partial T}+\frac{\partial\left(\rho V_{j}\right)}{\partial X_{j}}=0
$$

The statement of momentum conservation for the viscous fluid is

$$
\frac{\partial\left(\rho V_{i}\right)}{\partial T}=-\frac{\partial\left(\rho V_{i} V_{j}\right)}{\partial X_{j}}-\frac{\partial P_{i j}}{\partial X_{j}},
$$

where $P_{i j}$ is the compressive stress tensor defined as

$$
\begin{aligned}
P_{i j}= & P \delta_{i j}+\mu\left(-\frac{\partial V_{i}}{\partial X_{j}}-\frac{\partial V_{j}}{\partial X_{j}}+\frac{2}{3} \frac{\partial V_{k}}{\partial X_{k}} \delta_{i j}\right) \\
& +\mu_{B} \frac{\partial V_{k}}{\partial X_{k}} \delta_{i j},
\end{aligned}
$$

where $\mu$ is the shear viscosity, and $\mu_{B}$ is the bulk viscosity of the fluid. Under the assumption of isentropy the equation of state is

$$
\frac{D S_{e}}{D T}=0 \text {, }
$$

where $S_{e}$ is the entropy of the fluid.

Let us direct our attention to simplifying the mass conservation equation. Both the density and particle velocity of the gas are comprised of steady and time varying components. We can express the density and particle velocity in the $X_{i}$ direction as

$$
\begin{aligned}
& \rho=\bar{\rho}+[\rho-\bar{\rho}], \\
& V_{i}=\bar{V}_{i}+\left[V_{i}-\bar{V}_{i}\right],
\end{aligned}
$$

where the bracketed terms are time varying quantities and those headed with a bar are time averaged quantities. The momentum acting in $X_{i}$ direction is

$$
\rho V_{i}=\overline{\rho V_{i}}+\left(\rho V_{i}-\overline{\rho V_{i}}\right),
$$

where

$$
\overline{\rho V_{i}}=\bar{\rho} \bar{V}_{i}+\overline{(\rho-\bar{\rho})\left(V_{i}-\bar{V}_{i}\right)}
$$

and

$$
\begin{aligned}
\left(\rho V_{i}-\bar{\rho} \bar{V}_{i}\right)= & \bar{\rho}\left(V_{i}-\bar{V}_{i}\right)+(\rho-\bar{\rho}) \bar{V}_{i}+(\rho-\bar{\rho})\left(V_{i}-\bar{V}_{i}\right) \\
& -\overline{(\rho-\bar{\rho})\left(V_{i}-\bar{V}_{i}\right)} .
\end{aligned}
$$

As seen in Eq. (4), the time averaged momentum $\overline{\rho V_{i}}$ is the sum of the momentum of the streaming flow $\bar{\rho} \vec{V}_{i}$ and the time averaged oscillatory momentum $\overline{(\rho-\bar{\rho})\left(V_{i}-\bar{V}_{i}\right)}$. The time averaged oscillatory momentum is related to the sound field's time averaged acoustic intensity. Replacing $(\rho-\bar{\rho})$ with $(P-\bar{P}) / c^{2}$, the time averaged oscillatory momentum is

$$
\overline{(\rho-\bar{\rho})\left(V_{i}-\bar{V}_{i}\right)}=\overline{\left[(P-\bar{P})\left(V_{i}-\bar{V}_{i}\right)\right]} / c^{2}=\bar{I}_{i} / c^{2},
$$

where $\bar{I}_{i}$ is the $i$ th component of the time average acoustic intensity. We can also express the second-order part of the oscillatory momentum in Eq. (5) in terms of the instantaneous acoustic intensity:

$$
\left[(\rho-\bar{\rho})\left(V_{i}-\bar{V}_{i}\right)\right]-\left[\overline{(\rho-\bar{\rho})\left(V_{i}-\bar{V}_{i}\right)}\right]=\left(I_{i}-\bar{I}_{i}\right) / c^{2} .
$$

The time averaged density $\bar{\rho}$ will be taken to be approximately equal to the rest density $\rho_{0}$. The steady and unsteady momentum of the fluid can be rewritten as

$$
\overline{\rho V_{i}}=\rho_{0} V_{i}+\bar{I}_{i} / c^{2}
$$

and

$$
\left(\rho V_{i}-\overline{\rho V_{i}}\right)=\rho_{0}\left(V_{i}-\bar{V}_{i}\right)+\left(\rho-\rho_{0}\right) \bar{V}_{i}+\left(I_{i}-\bar{I}_{i}\right) / c^{2} .
$$

Under the assumptions

$$
\frac{\partial}{\partial X_{j}}\left[\left(\rho-\rho_{0}\right) V_{j}\right] \simeq 0
$$

or equivalently

$$
\frac{\partial}{\partial X_{j}}\left[I_{j} / c^{2}+\left(\rho-\rho_{0}\right) \bar{V}_{j}\right] \simeq 0
$$

and

$$
\bar{\rho} \sim \rho_{0},
$$


the simplified statement of mass conservation is

$$
\frac{\partial \rho}{\partial T}+\rho_{0} \frac{\partial V_{j}}{\partial X_{j}} \simeq 0
$$

We see that in Eq. (6) we have the divergence of the sum of two terms being set equal to zero. The first term in the expression is proportional to the loss of acoustic power per unit volume. The divergence of the acoustic intensity for a plane acoustic wave is

$$
O\left[U_{0}^{2}(\omega / c)^{2}\left(\frac{4}{3} \mu+\mu_{B}\right)\right] .
$$

If the frequency of excitation is sufficiently low the loss of acoustic power per unit volume is small. For this reason the mass flux contributed by the divergence of the acoustic intensity will be neglected in our analysis. Hence, streaming generated by the loss of acoustic energy in the bulk will not be addressed in this paper. The second term then becomes $\nabla\left(\rho-\rho_{0}\right) \cdot \bar{\nabla}$. We will assume that the divergence of $\left(\rho-\rho_{0}\right) \bar{V}$ is small when compared to the time rate of change of the density. Therefore, in using Eq. (7) we have bounded the amplitude range of investigation and have limited ourselves to problems in acoustic streaming where boundary losses play the major role. We can simplify the momentum equation in a more direct manner. Substituting the exact statement of mass conservation, Eq. (1), into the momentum equation (2) yields

$$
\rho \frac{\partial V_{i}}{\partial T}+\rho\left(V_{j} \frac{\partial V_{i}}{\partial X_{j}}\right)=-\frac{\partial P_{i j}}{\partial X_{j}} .
$$

Under the assumption that

$$
\rho_{0}>\left|\rho-\rho_{0}\right|,
$$

the momentum equation can be rewritten as

$$
\rho_{0} \frac{\partial V_{i}}{\partial T}+\rho_{0} V_{j} \frac{\partial V_{i}}{\partial X_{j}} \simeq \frac{\partial P_{i j}}{\partial X_{j}} .
$$

The approximate set of 'equations which satisfy assumptions made in Eqs. (6) and (9) written in vector form are

$\frac{1}{\rho_{0} c^{2}} \frac{\partial P}{\partial T}+\nabla \cdot \mathrm{V}=0$

$\rho_{0} \frac{\partial \mathbf{V}}{\partial T}+\rho_{0} \mathbf{V} \cdot \nabla \mathbf{V}+\nabla P=\mu \nabla^{2} \mathbf{V}+\left[\left(\frac{1}{3} \mu+\mu_{B}\right) \nabla \nabla \cdot \mathbf{V}\right]$

Using Eq. (11) we can rewrite the bracketed term in Eq. (12) as

$$
\left[\left(\frac{1}{3} \mu+\mu_{B}\right) \frac{1}{\rho_{0} c^{2}} \frac{\partial \nabla P}{\partial T}\right] .
$$

The bracketed term is responsible for acoustic streaming in the presence of bulk losses. Eckhart ${ }^{9}$ has shown the amplitude of the streaming velocity due to bulk losses is proportional to $(\omega / c)^{2}\left(\frac{4}{3} \mu+\mu_{B}\right) U_{0}^{2}$, where $U_{0}$ is the typical amplitude oscillatory particle velocity. The streaming velocity resulting from boundary losses ${ }^{1}$ is proportional to $U_{0}^{2} / \omega$. Therefore for sufficiently low frequencies we can assume that

$$
\nabla P>\left[\left(\frac{1}{3} \mu+\mu_{B}\right) \frac{1}{\rho_{0} c^{2}} \frac{\partial \nabla P}{\partial T}\right] .
$$

Applying conditions given in Eqs. (6), (9), and (13) to Eqs. (1)-
(3) we obtain a set of approximate equations:

$$
\begin{aligned}
& \rho_{0} \frac{D \mathrm{~V}}{D T}+\nabla P=\mu \nabla^{2} \mathrm{~V}, \\
& \frac{1}{\rho_{0} c^{2}} \frac{\partial P}{\partial T}+\nabla \cdot \mathrm{V}=0,
\end{aligned}
$$

where

$$
\left.c^{2} \equiv \frac{\partial P}{\partial \rho}\right|_{s_{e}=\text { const }} .
$$

We will now decompose the single set of equations given in Eq. (14) into two sets. The first will govern the time average motion of the fluid and the second the unsteady motion. Taking the time average of Eq. (14) yields the equations which govern the time average motion of the fluid

$$
\begin{aligned}
& \rho_{0}(\overline{\mathbf{V} \cdot \nabla \mathbf{V}})+\nabla \bar{P}=\mu \nabla^{2} \bar{V}, \\
& \nabla \cdot \bar{V}=0,
\end{aligned}
$$

where

$$
(\overline{\mathbf{V} \cdot \nabla \mathbf{V}})=[(\overline{\mathbf{V}-\overline{\mathbf{V}}) \cdot \nabla(\mathbf{V}-\overline{\mathbf{V}}})+\overline{\mathbf{V}} \cdot \nabla \overline{\mathbf{V}}] .
$$

Subtracting the result given in Eq. (15) from Eq. (14) yields the equations governing the unsteady motion of the fluid:

$$
\begin{aligned}
& \frac{\rho_{0} \partial(\mathbf{V}-\overline{\mathbf{V}})}{\partial T}+\rho_{0}(\mathbf{V} \cdot \nabla \mathbf{V}-\overline{\mathbf{V} \cdot \nabla \mathbf{V}})+\nabla(P-\bar{P}) \\
& \quad=\mu \nabla^{2}(\mathbf{V}-\overline{\mathbf{V}}) \\
& \frac{1}{\rho_{0} c^{2}} \frac{\partial(P-\bar{P})}{\partial T}+\nabla \cdot(\mathbf{V}-\overline{\mathbf{V}})=0 \\
& \text { where }
\end{aligned}
$$

$$
\begin{aligned}
(\mathbf{V} \cdot \nabla \mathbf{V} & -\overline{\mathbf{V} \cdot \nabla \mathbf{V}}) \\
= & {[(\mathbf{V}-\overline{\mathbf{V}}) \cdot \nabla(\mathbf{V}-\overline{\mathbf{V}})-(\overline{\mathbf{V}-\overline{\mathbf{V}}) \cdot \nabla(\mathbf{V}-\overline{\mathbf{V}})}} \\
& +(\mathbf{V}-\overline{\mathbf{V}}) \cdot \nabla \overline{\mathbf{V}}+\overline{\mathbf{V}} \cdot \nabla(\mathbf{V}-\overline{\mathbf{V}})] .
\end{aligned}
$$

\section{NONDIMENSIONALIZATION OF THE EQUATIONS OF MOTION}

In this section we will present a scheme for nondimensionalizing the equations of motion. The resultant nondimensional set of equations will govern the acoustic wave propagation and streaming in a duct with slowly varying height.

Consider a two-dimensional waveguide having a height which varies slowly with $X$ (see Fig. 1). The lower wall is located at $Y=0$ and location of the upper wall is given by the function $Y=H_{0} h\left(X / L_{0}\right) \cdot H_{0}$ is the typical height of the duct and $L_{0}$ represents the characteristic wall wavelength. The acoustic field in the waveguide is produced by two traveling pressure waves which vary harmonically with time at frequency $\omega$. One wave is launched at $X=-X_{0}$ in the positive $X$ direction and the other is launched in the negative $X$ direction at $X=X_{1}$. The dynamic behavior of the fluid is characterized by the relationship among five length scales, namely:

(1) the acoustic wavelength $\lambda$,

(2) the wall wavelength $L_{0}$, 
(3) the typical wall height $H_{0}$,

(4) the oscillatory boundary layer thickness $l_{v}$,

(5) the oscillatory particle displacement $U_{0} / \omega$.

We will assume that $L_{0} / \lambda=O(1)$ and $H_{0} h\left(X / L_{0}\right)=O\left(H_{0}\right)$ for all $X$. For only the principal mode to propagate the condition

$$
\left(\omega L_{0} / c\right)\left(H_{0} / L_{0}\right)<1
$$

or equivalently

$$
\epsilon k<1 \text {, }
$$

where $\epsilon=H_{0} / L_{0}$ is of the order of the slope of the upper wall and is a small parameter, and $k=\omega / c L_{0}$ is the nondimensional wavenumber, must be satisfied. The slope of the upper wall is assumed to be $O(\epsilon)$. Therefore,

$$
\frac{\partial H\left(X / L_{0}\right)}{\partial X}=\frac{H_{0}}{L_{0}} \frac{\partial h\left(X / L_{0}\right)}{\partial\left(X / L_{0}\right)}=\epsilon h^{\prime}\left(X / L_{0}\right)=O(\epsilon) .
$$

Both duct walls are assumed to be rigid. Therefore, at $Y=0$ and $Y=H_{0} h\left(X / L_{0}\right)$ the normal and tangential oscillatory particle velocities equal zero. Given that the upper boundary varies slowly with $X$, a simple relationship between the vertical and horizontal oscillatory particle velocities can be given. For the normal velocity to be zero on the upper wall the resultant vector velocity must be parallel to the line tangent to $Y=H_{0} h\left(X / L_{0}\right)$. Therefore, the typical amplitude of the $Y$ component of the vector velocity $V_{0}$, must be related to the typical horizontal component $U_{0}$ as $V_{0}=O\left[U_{0}\left(H_{0} d h / d X\right)\right]$. Given that the wall slope is $O(\epsilon)$ we expect the typical vertical velocity $V_{0}$ to be $O\left(\epsilon U_{0}\right)$. Hence

$$
V_{0}=\epsilon U_{0},
$$

where $V_{0}$ represents the typical oscillatory particle velocity in the vertical direction, $U_{0}$ represents the typical oscillatory particle velocity in the horizontal direction, and $\epsilon$ represents the typical wall slope. The previous statement relates the two oscillatory amplitudes, $U_{0}$ and $V_{0}$ to the wall slope.

Let us now consider the relationship between the vertical and horizontal components of the steady velocity. The streaming velocity of the fluid was driven by the gradient of the time average Reynolds stress. The typical amplitude of the steady horizontal velocity is $O\left[\epsilon U_{0}^{2} /\left(H_{0} \omega\right)\right]$. The typical horizontal streaming velocity amplitude $\bar{U}_{0}$ will be set equal to $U_{0}^{2} /\left(H_{0} \omega\right)$. The streaming velocity of the fluid in the vertical direction is implicitly dependent on the transverse wave number, $\omega / c H_{0}=k \epsilon$. We expect the typical vertical streaming velocity amplitude $\bar{V}_{0}$ is $O\left[U_{0}^{2} \epsilon /\left(H_{0} \omega\right)\right]$.

The typical convection time $H_{0} / U_{0}$ of the streaming velocity is large compared to the period of oscillation. Hence, we will express fluctuations in time using a long time scale. We will set the nondimensional time $t$ equal to $\omega T$. is

Our nondimensionalization for the oscillatory variables

$$
\begin{aligned}
& u=U / U_{0}, \\
& v=V / V_{0}=V /\left(U_{0} \epsilon\right), \\
& p=P /\left(\omega \rho_{0} L_{0} U_{0}\right), \\
& t=\omega T, \\
& k=\omega / c L_{0} .
\end{aligned}
$$

For the steady variables, the nondimensionalization is

$$
\begin{aligned}
& \bar{u}=\bar{U} /\left[U_{0}^{2} /\left(\omega H_{0}\right)\right], \\
& \bar{v}=\bar{V} /\left[U_{0}^{2} \epsilon /\left(\omega H_{0}\right)\right], \\
& \bar{p}=\bar{P} /\left[\omega \rho_{0} L_{0} U_{0}\right],
\end{aligned}
$$

and for the coordinates

$$
\begin{aligned}
& x=X / L_{0}, \\
& y=Y / H_{0} .
\end{aligned}
$$

Substituting our nondimensionalized variables into the set of approximate equations, given in Sec. I, yields for the unsteady momentum acting in $x$

$$
\begin{aligned}
\dot{u}+ & (\epsilon / S)\left(u u_{x}+v u_{y}-\overline{u u_{x}}-\overline{v u_{y}}\right)+\left(\epsilon / S^{2}\right) \\
& \times\left[(\bar{u} u)_{x}+\bar{v} u_{y}+\bar{u}_{y} v\right]+P_{x}=(1 / R)\left(u_{y y}+\epsilon^{2} u_{x x}\right),
\end{aligned}
$$

in $y$

$$
\begin{aligned}
\epsilon^{2} \dot{v}+ & \left(\epsilon^{3} / S\right)\left(u v_{x}+v v_{y}-\overline{u v_{x}}-\overline{v v_{y}}\right)+\left(\epsilon^{3} / S^{2}\right) \\
& \times\left[\bar{u} v_{x}+\bar{v}_{x} u+(\bar{v} v)_{y}\right]+p_{y}=\left(\epsilon^{2} / R\right)\left(v_{y y}+\epsilon^{2} v_{x x}\right),
\end{aligned}
$$

and the unsteady continuity equation is

$$
u_{x}+v_{y}=-k^{2} \dot{p},
$$

where $S$ is the Strouhal number, $\omega H_{0} / U_{0}, R$ is the oscillatory Reynolds number, $\omega H_{0}^{2} / v=H_{0}^{2} / l_{v}^{2}$, and $\epsilon=H_{0} / L_{0}$.

The nondimensional equation governing the steady momentum in $x$ is

$$
\begin{gathered}
(\epsilon / S)\left(\overline{u u_{x}}+\overline{v u_{y}}\right)+\left(\epsilon / S^{3}\right)\left(\bar{u} \bar{u}_{x}+\bar{v} \bar{u}_{y}\right)+\bar{p}_{x} \\
=(1 / R S)\left(\bar{u}_{y y}+\epsilon^{2} \bar{u}_{x x}\right),
\end{gathered}
$$

in $y$ is

$$
\begin{gathered}
\left(\epsilon^{3} / S\right)\left(\overline{u v_{x}}+\overline{v v_{y}}\right)+\left(\epsilon^{3} / S^{3}\right)\left(\bar{u} \bar{v}_{x}+\overline{v v}_{y}\right)+\bar{p}_{y} \\
=\left(\epsilon^{2} / R S\right)\left(\bar{v}_{y y}+\epsilon^{2} \bar{v}_{x x}\right),
\end{gathered}
$$

and the steady continuity equation is

$$
\bar{u}_{x}+\bar{v}_{y}=0 \text {. }
$$

The behavior of the fluid in the duct is characterized by the values of three nondimensional parameters, $S, R$, and $\epsilon$. Each of these parameters can be interpreted physically. $\epsilon$ is the wall slope, $S$ is the ratio of the characteristic height of the guide to the oscillatory displacement, and $R$ is the ratio of the linear oscillatory internal force to the linear viscous force acting in the horizontal direction, or the square of the ratio of the typical height of the guide to the viscous length $l_{v}$.

We wish to determine the solution in the limiting case of $1 / S, 1 / R$, and $\epsilon$ going to zero. In the duct we will determine the solution in terms of an asymptotic sequence in $\epsilon$, holding $1 / S$ and $1 / R$ small and fixed. Since the equations are regular in $\epsilon$ we expect the solution to be regular in the limit as $1 / S$ and $1 / R$ tends to zero.

\section{THE ZEROTH COEFFICIENT}

The zeroth order equations of oscillatory motion are

$$
\begin{aligned}
& \dot{u}_{0}+p_{0_{x}}=1 / R u_{0_{y y}}, \\
& p_{0_{y}}=0, \\
& u_{0_{x}}+v_{0_{y}}=-k^{2} \dot{p}_{0},
\end{aligned}
$$


and

$$
\begin{aligned}
& \bar{p}_{0_{x}} \doteq 1 /(R S) \bar{u}_{0_{y y}}, \\
& \bar{p}_{0_{y}}=0, \\
& \bar{u}_{0_{x}}+\bar{v}_{0_{y}}=0,
\end{aligned}
$$

for the steady motion of the fluid where

$$
u_{0}=v_{0}=\bar{u}_{0}=\bar{v}_{0}=0
$$

on $y=0$ and $y=h(x)$.

The amplitude of the steady velocity $\bar{u}_{0}$ is independent of the oscillatory motion of the fluid in the duct. In the absence of discontinuities in the guide walls, there are no sources that drive the steady motion of the fluid at this order of approximation. Therefore,

$$
\bar{u}_{0}=\bar{v}_{0}=\bar{p}_{0_{x}}=0 \text {. }
$$

Since $p_{0_{y}}$ equals zero, the oscillatory pressure $p_{0}$ is only a function of $x$ and $t$. The complex amplitude of $u_{0}$ is evaluated in terms of the pressure $p_{0}$ using Eq. (23). The vertical velocity $v_{0}$ is obtained by integrating the continuity equation with respect to $y$. This integration yields one arbitrary constant which is used to satisfy the boundary condition that $v_{0}=0$ on $y=0$. In order to satisfy the boundary condition that $v_{0}$ equal zero on the upper wall of the guide, we will show that the pressure is constrained to be a solution of the lossy Webster horn equation.

Solving for $u_{0}$ in Eq. (23) and applying the boundary condition that $u_{0}$ equals zero at $y=0$ and $y=h(x)$ we obtain the result

$$
\begin{aligned}
u_{0}= & \operatorname{Re}\left\{-i p_{0}^{\prime}\left[1-e^{-A y}\left(\frac{1-e^{-A h}}{1-e^{-A 2 h}}\right)\right.\right. \\
& \left.\left.-e^{-A(h-y)}\left(\frac{1-e^{-A h}}{1-e^{-A h 2}}\right)\right] e^{-i t}\right\},
\end{aligned}
$$

where

$$
A=(1-i) \alpha,
$$

and

$$
\alpha=(R / 2)^{1 / 2} .
$$

Under the assumption that the Reynolds number is large the horizontal component of the oscillatory particle velocity can be approximated as

$$
u_{0}=\operatorname{Re}\left[-i p_{0}^{\prime}\left(1-e^{-A h}-e^{-A(h-y)}\right) e^{-i t}\right]+O\left(e^{-\alpha h}\right) .
$$

We can see by this expression that the error introduced in our approximation is exponentially small for large values of the Reynolds number. The zeroth order solutions for the steady pressure and velocity are

$$
\begin{aligned}
\bar{u}_{0} & =\bar{v}_{0}=0, \\
\bar{p}_{0} & =a \text { constant } \\
u_{0} & =\operatorname{Re}\left[i p_{0}^{\prime}\left(1-e^{-A y}-e^{-A(h-y)}\right) e^{-i t}\right]+O\left(e^{-\alpha h}\right),
\end{aligned}
$$

and

$$
\begin{aligned}
v_{0}= & \operatorname{Re}\left\{\left[i w_{0} y+i p_{0}^{\prime \prime}\left(e^{-A y}-e^{-A(h-y)}-1\right) / A\right.\right. \\
& \left.\left.+i p_{0}^{\prime} h^{\prime} e^{-A(h-y)}\right] e^{-i t}\right\}+O\left(e^{-\alpha h}\right),
\end{aligned}
$$

where

$$
\begin{aligned}
& A=\alpha(1-i), \\
& \alpha=(R / 2)^{1 / 2}=\left[H_{0}^{2} \omega /(2 v)\right]^{1 / 2}, \\
& w_{0}=p_{0}^{\prime \prime}+k^{2} p_{0}, \\
& p_{0}=p_{0}(x, k), \\
& h=h(x) .
\end{aligned}
$$

The expression for the vertical velocity given in Eq. (32) will satisfy the boundary condition that $v_{0}=0$ on $y=h(x)$ only if the pressure satisfies the equation

$$
\left(h p_{0}^{\prime}\right)^{\prime}+h k^{2} p_{0}-2 p_{0}^{\prime \prime} / A+O\left(e^{-\alpha h}\right)=0 .
$$

Therefore the pressure is constrained to be a solution of the lossy Webster horn equation.

\section{THE FIRST-ORDER COEFFICIENT}

By equating the like terms in $\epsilon$ and using the result $\bar{u}_{0}=0$ and $\bar{v}_{0}=0$, the first-order equations can be determined. The equations that govern the oscillatory motion of the fluid are

$$
\begin{aligned}
& \dot{u}_{1}+(1 / S)\left(u_{0} u_{0_{x}}+v_{0} u_{0_{y}}-\overline{u_{0} u_{0_{x}}}-\overline{v_{0} u_{0_{y}}}\right)+p_{1_{x}} \\
& \quad=(1 / R) u_{1_{y y}}, \\
& p_{1_{y}}=0 \\
& u_{1_{x}}+v_{1_{y}}=-k^{2} \dot{p}_{1},
\end{aligned}
$$

and

$$
\begin{aligned}
& (1 / S)\left(\overline{u_{0} u_{0_{x}}}+\overline{v_{0} u_{0_{y}}}\right)+\bar{p}_{1_{x}}=(1 / R S) \bar{u}_{1_{y y}}, \\
& \bar{p}_{1_{y}}=0,
\end{aligned}
$$

and

$$
\bar{u}_{1_{x}}+\bar{v}_{1_{y}}=0
$$

govern the steady motion.

As in the case of the zeroth-order pressures, $\bar{p}_{1}$ and $p_{1}$ are independent of $y$. Using the solutions for the oscillatory velocities, $u_{0}$ and $v_{0}$, we can evaluate the driving term in Eq. (37). The streaming velocity $\bar{u}_{1}$ is determined by integrating the steady momentum equation twice with respect to $y$ and applying the zero velocity condition on the boundaries. The resulting expression is functionally dependent on the amplitudes of $\bar{p}_{1}, p_{0}, h$, and their derivatives. The result for $\bar{u}_{1}$, neglecting exponentially small terms, is

$$
\begin{aligned}
\bar{u}_{1}= & \left(\frac{R S}{2} \bar{p}_{1}^{\prime}+\frac{R}{2} A_{1}\right)\left(y^{2}-h y\right)+A_{1}\left[\phi_{1_{y}}-\left(\frac{3}{2}-\frac{2}{h \alpha}\right)\right] \\
& -A_{2}\left[\phi_{2_{y}}-\left(\frac{-1}{2}+\frac{2}{h \alpha}\right)\right]+A_{3} \phi_{3_{y}}+O\left(e^{-\alpha h}\right),
\end{aligned}
$$

where

$$
\begin{aligned}
& A_{1}=\operatorname{Re}\left(p_{0}^{* \prime} p_{0}^{\prime \prime}\right) / 2, \\
& A_{2}=\operatorname{Im}\left(p_{0}^{* \prime} p_{0}^{\prime \prime}\right) / 2, \\
& A_{3}=\left|p_{0}^{\prime}\right|^{2} / 2,
\end{aligned}
$$




$$
\begin{aligned}
& \phi_{1_{y}}=e^{-\alpha y}\left[2 \sin (\alpha y)+\cos (\alpha y)+e^{-\alpha y} / 2\right]+e^{-\alpha y}\left[2 \sin (\alpha \tilde{y})-\cos (\alpha \tilde{y})+e^{-\alpha \tilde{y}} / 2\right]+(2 / \alpha h)\left\{e^{-\alpha y}[-\cos (\alpha y)+\sin (\alpha y)]\right\} \\
& +(2 / \alpha h)\left\{e^{-\alpha h}[-\cos (\alpha \tilde{y})+\sin (\alpha \tilde{y})]\right\}-(2 / h) y\left[e^{-\alpha y} \cos (\alpha y)-\cos (\alpha \tilde{y}) e^{-\alpha \tilde{y}}\right], \\
& \phi_{2_{y}}=e^{-\alpha y}\left[\sin (\alpha y)+e^{-\alpha y} / 2\right]+e^{-\alpha \tilde{y}}\left[-\sin (\alpha \tilde{y})+e^{-\alpha \tilde{y}} / 2\right]+(2 / \alpha h)\left\{e^{-\alpha y}[\cos (\alpha y)+\sin (\alpha y)]+e^{-\alpha y}[\cos (\alpha \tilde{y})+\sin (\alpha \tilde{y})]\right\} \\
& +(2 / h) y\left[e^{-\alpha y} \sin (\alpha y)-e^{-\alpha \bar{y}} \sin (\alpha \tilde{y})\right], \\
& \phi_{3_{y}}=\tilde{y} \frac{\alpha h^{\prime}}{h} e^{-\alpha \bar{y}}[\cos (\alpha \tilde{y})-\cos (\alpha \tilde{y})]+y \frac{\alpha h^{\prime}}{h} e^{-\alpha y}[\cos (\alpha y)-\sin (\alpha y)]-2 \frac{h^{\prime}}{h}\left[e^{-\alpha y} \sin (\alpha y)+e^{-\alpha \bar{y}} \sin (\alpha \tilde{y})\right], \\
& \alpha=(R / 2)^{1 / 2} \text {, } \\
& \tilde{y}=h-y \text {, }
\end{aligned}
$$

and

$$
h=h(x) \text {. }
$$

The variable $\bar{p}_{1}$ must be determined by first solving for $\bar{v}_{1}$ and applying the boundary conditions to the result. The first bracketed expression in Eq. (40)

$$
(R S / 2)\left(\bar{p}_{1}^{\prime}\right)+(R / 2) A_{1},
$$
where $R>1$, initially appears to be $O(R)$ or $O(R S)$. However, we will see later that this term is actually of $O(1)$. In $\phi_{3_{y}}$ we see
terms which behave like

$$
h^{\prime} \alpha y e^{-\alpha y},
$$

where $\alpha>1$. The maximum value of this term occurs when $y=1 / \alpha$. Therefore, the solution at this stage is uniformly valid in $y$.

The mean vertical velocity $\bar{v}_{1}$ is determined by integrating Eq. (39) with respect to $y$ and applying the zero velocity boundary conditions. When the boundary conditions are applied, a single inhomogeneous differential equation is obtained. This equation relates the static pressure $\bar{p}_{1}$ to terms involving Reynolds stress at the upper wall and must be satisfied if the $\bar{v}_{1}$ is to be equal to zero on the upper and lower walls of the guide. The vertical component of the streaming velocity is

$$
\begin{aligned}
\bar{v}_{1}= & -\left[\left(\frac{R S}{2}\left(\bar{p}_{1}^{\prime}\right)+\frac{R}{2} A_{1}\right)\left(\frac{y^{3}}{3}-\frac{h y^{2}}{2}\right)\right]_{x}-\left\{A_{1}\left[\phi_{1}-\left(\frac{3}{2}-\frac{2}{h \alpha}\right) y\right]_{x}\right\}-\left\{A_{2}\left[\phi_{2}-\left(\frac{-1}{2}+\frac{2}{h \alpha}\right) y\right]_{x}\right\} \\
& -\left[A_{3}\left(\phi_{3}-\frac{h^{\prime} 3}{h \alpha 2}\right)\right]_{x}-A_{3} \frac{k^{2}}{2 \alpha}\left(\phi_{4}-1\right)+O\left(e^{-\alpha h}\right),
\end{aligned}
$$

where

$$
\begin{aligned}
\frac{R S}{2} & \bar{p}_{1}^{\prime}+\frac{R}{2} A_{1}=-A_{1}\left(\frac{9}{h^{2}}-\frac{21}{\alpha h^{3}}\right)-A_{2}\left(\frac{36}{h^{4} \alpha^{2}}+\frac{3}{h^{2}}-\frac{21}{\alpha h^{3}}\right)-A_{3}\left(\frac{h^{\prime}}{h^{4} \alpha} 18\right), \\
\phi_{1}= & \frac{1}{2 \alpha}\left[e^{-\alpha y}\left(-\sin (\alpha y)-3 \cos (\alpha y)-\frac{e^{-\alpha y}}{2}\right)\right]+\frac{1}{2 \alpha}\left[e^{-\alpha \tilde{y}}\left(3 \sin (\alpha \tilde{y})+\cos (\alpha \tilde{y})+\frac{e^{-\alpha \tilde{y}}}{2}\right)\right] \\
& +\frac{3}{h \alpha^{2}}\left[e^{-\alpha \tilde{y}} \sin (\alpha \tilde{y})-e^{-\alpha y} \sin (\alpha y)\right]+\frac{1}{h \alpha} y\left\{e^{-\alpha y}[-\cos (\alpha y)+\sin (\alpha y)]+e^{-\alpha \tilde{y}}[-\cos (\alpha \tilde{y})+\sin (\alpha \tilde{y})]\right\}, \\
\phi_{2}= & \frac{1}{2 \alpha}\left[e^{-\alpha y}\left(\sin (\alpha y)+\cos (\alpha y)+\frac{e^{-\alpha y}}{2}\right)\right]+\frac{1}{2 \alpha}\left[e^{-\alpha \tilde{y}}\left(\sin (\alpha \tilde{y})+\cos (\alpha \tilde{y})-\frac{e^{-\alpha \tilde{y}}}{2}\right)\right] \\
& +\frac{1}{h \alpha^{2}}\left[-3 e^{-\alpha y} \cos (\alpha y)+3 e^{-\alpha \bar{y}} \cos (\alpha \tilde{y})\right]+\left(\frac{1}{2 h \alpha^{3}}\right) y\left\{e^{-\alpha y}[\cos (\alpha y)+\sin (\alpha y)]+e^{-\alpha \tilde{y}}[\cos (\alpha \tilde{y})+\sin (\alpha \tilde{y})]\right\}, \\
\phi_{3}= & \frac{-h^{\prime}}{2 \alpha^{2}} e^{-\alpha \tilde{y}} \sin (\alpha \tilde{y})-\frac{h^{\prime}}{h 2 \alpha}\left\{-3 e^{-\alpha y}[\sin (\alpha y)+\cos (\alpha y)]\right. \\
& \left.+3 e^{-\alpha \tilde{y}}[\sin (\alpha \tilde{y})+\cos (\alpha \tilde{y})]\right\}+\frac{h^{\prime}}{h} y\left[e^{-\alpha y} \sin (\alpha y)+e^{-\alpha \tilde{y}} \sin (\alpha \tilde{y})\right],
\end{aligned}
$$

and

$$
\phi_{4}=\left\{e^{-\alpha y}[\cos (\alpha y)+\sin (\alpha y)]-e^{-\alpha \tilde{y}}[\cos (\alpha \tilde{y})+\sin (\alpha \tilde{y})]\right\} .
$$

Outside the boundary layer the expressions given for the streaming flow greatly simplify. Taking the limit $\bar{u}_{1}$ and $\bar{v}_{1}$ as $a \rightarrow \infty$ while holding $y$ fixed yields

$\bar{u}_{1}=-\left(A_{1} \frac{3}{2}+A_{2} \frac{1}{2}\right)\left[1+6\left(\frac{y^{2}}{h^{2}}-\frac{y}{h}\right)\right]+O\left(\frac{1}{\alpha}\right)$,

$$
\begin{aligned}
& \bar{v}_{1}=\left\{\left(A_{1} \frac{3}{2}+A_{2} \frac{1}{2}\right)\left[y+6\left(\frac{y^{3}}{3 h^{2}}-\frac{y}{2 h}\right)\right]\right\}_{x}+O\left(\frac{1}{\alpha}\right), \\
& \bar{\psi}_{1}=-\left(A_{1} \frac{3}{2}+A_{2} \frac{1}{2}\right)\left[y+6\left(\frac{y^{3}}{3 h^{2}}-\frac{y^{2}}{2 h}\right)\right]+O\left(\frac{1}{\alpha}\right) .
\end{aligned}
$$

In order to obtain a more physical picture of the streaming 
phenomena, we will assume a simple form for the zero-order oscillatory pressure field. Say the zeroth-order pressure field can be represented as a sum of an incident and a reflected wave:

$$
\begin{aligned}
& p_{0}(x, k, t)=\operatorname{Re}\left[p_{0}(x, k) e^{-i t}\right], \\
& p_{0}(x, k, t)=\operatorname{Re}\left[\tilde{A}\left(e^{+i r}+\left|R_{f}\right| e^{-i\left(r^{*}-\theta\right)}\right) e^{-i t}\right],
\end{aligned}
$$

where $\tilde{A}$ is the pressure amplitude of the incoming wave, $\boldsymbol{R}_{f}=\left|\boldsymbol{R}_{f}\right| e^{+i \theta}$ is the reflection coefficient, and $\gamma=\gamma(x, k, \alpha)$ is the phase function. If the losses at the walls are small, we can neglect their effect on the phase function $\gamma$. Thus

$$
\gamma=\gamma(x, k)
$$

is a real function when $k$ is greater than $k_{0}$, where $k_{0}$ represents the cutoff wavenumber of the guide.

Using Eq. (44), the real and imaginary parts of $p_{0}^{* \prime} p_{0}^{\prime \prime}$ can be tabulated:

$$
\begin{aligned}
& \operatorname{Re}\left(p_{0}^{* \prime} p_{0}^{\prime \prime}\right)=\widetilde{A}^{2}\left[2 \gamma^{\prime 2}\left|R_{f}\right| \sin (2 \gamma-\theta)+\gamma^{\prime} \gamma^{\prime \prime}\left(1-\left|R_{f}\right|^{2}\right)\right], \\
& \operatorname{Im}\left(p_{0}^{* \prime} p_{0}^{\prime \prime}\right)=\tilde{A}^{2} \gamma^{\prime 3}\left(1-\left|R_{f}\right|^{2}\right) .
\end{aligned}
$$

The real part of $p_{0}^{* \prime} p_{0}^{\prime \prime}$ is made up of two terms. The first is linearly dependent on the magnitude of the reflection coefficients $\boldsymbol{R}_{f}$ and is modulated sinusoidally at twice $\gamma$. The amplitude of the second term is proportional to $\left(1-\left|R_{f}\right|^{2}\right)$ and its spatial variation is dependent on the product of the first and second derivatives of the phase function. However, $\gamma^{\prime \prime}$ is usually small in a smooth duct. The amplitude of the imaginary part of $p_{0}^{* \prime} p_{0}^{\prime \prime}$ is proportional to $\left(1-\left|R_{f}\right|^{2}\right)$ and its spatial variation is dependent on $\left(\gamma^{\prime}\right)^{3}$.

The term $\left(1-\left|R_{f}\right|^{2}\right)$ is proportional to the difference between the mean vector acoustic intensity of the incoming wave and that of the reflected wave. If $\left|R_{f}\right|$ is equal to 1 , we would be left with

$\bar{u}_{1} \sim \tilde{A}^{2} \frac{3}{2} \gamma^{\prime 2} \sin (2 \gamma-\theta)\left[1+6\left(\frac{y^{2}}{h^{2}}-\frac{y}{h}\right)\right]+O\left(\frac{1}{\alpha}\right)$

and

$$
\begin{aligned}
\bar{v}_{1} \sim \tilde{A}^{2} & \frac{3}{2}\left\{\left[\gamma^{\prime 2} \sin (2 \gamma-\theta)\right]\right\}\left[y+6\left(\frac{y^{3}}{3 h^{2}}-\frac{y^{2}}{2 h}\right)\right]_{x} \\
+ & O\left(\frac{1}{\alpha}\right)
\end{aligned}
$$

in this case. If the slope of the upper wall, $h^{\prime}$, was set equal to zero, $\gamma^{\prime}$ would equal the nondimensional wavenumber, $k$. The resulting expression is identical to that obtained by Rayleigh in 1883. Acoustic streaming motion in the duct will take the form of standing vortices (see Fig. 2). On the other hand if $R_{f}$ equals zero, the stream velocities are

$\bar{u}_{1} \sim-\frac{\tilde{A}^{2}}{4}\left[3 \gamma^{\prime} \gamma^{\prime \prime}+\left(\gamma^{\prime}\right)^{3}\right]\left[1+6\left(\frac{y^{2}}{h^{2}}-\frac{y}{h}\right)\right]+O\left(\frac{1}{\alpha}\right)$

and

$$
\begin{aligned}
\bar{v}_{1} \sim & \frac{\widetilde{A}^{2}}{4}\left\{\left[3 \gamma^{\prime} \gamma^{\prime \prime}+\left(\gamma^{\prime}\right)^{3}\right]\left[y+6\left(\frac{y^{3}}{3 h^{2}}-\frac{y^{2}}{2 h}\right)\right]\right\}_{x} \\
+ & O\left(\frac{1}{\alpha}\right) .
\end{aligned}
$$

If $3 \gamma^{\prime} \gamma^{\prime \prime}+\left(\gamma^{\prime}\right)^{3}$ has no zeros, for all $x$, the streaming flow will

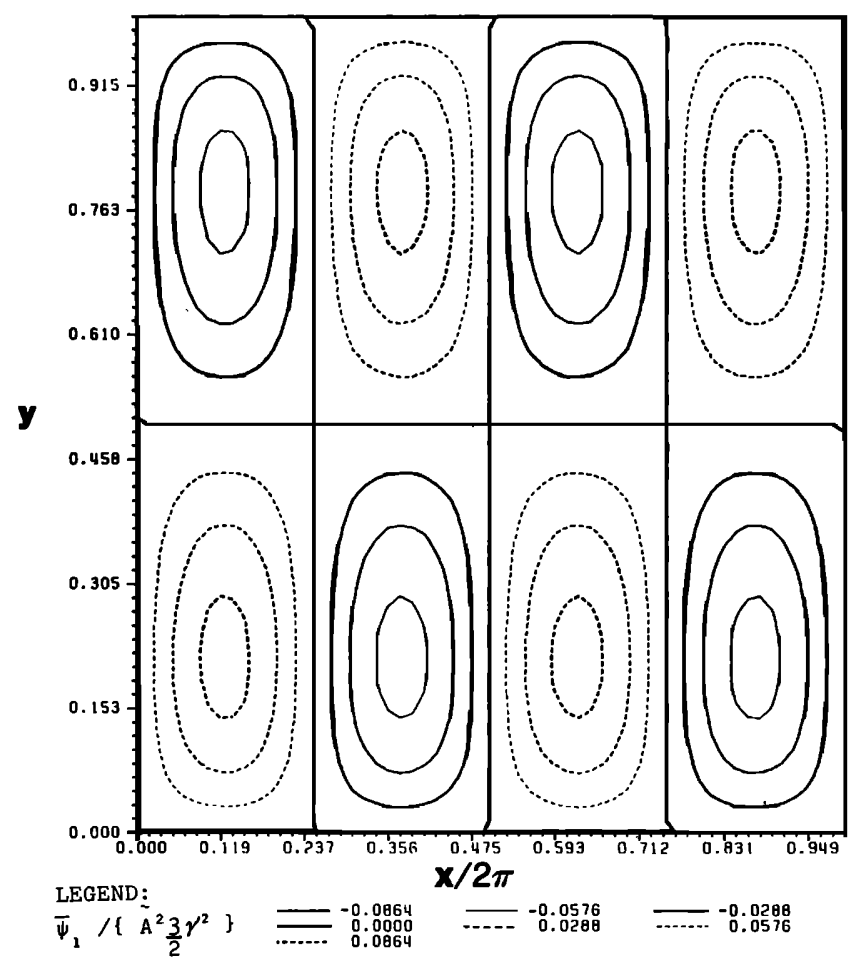

FIG. 2. Streaming motion in a duct having uniform height $h=1$ with $R_{f}=1$ and $\gamma^{\prime}=2 \pi$. Constant values of $\vec{\psi}_{1} /\left(\widetilde{A}^{2}{ }^{2} \gamma^{\prime 2}\right)$ are plotted versus $x / 2 \pi$ and $y$.

be jetlike (see Fig. 3). There is a steady outflow directed along the centerline of the duct and an inflow at the duct boundaries.

The aforementioned result seems to be in direct opposition to that reported by Eckart" who stated "when the sound beam fills the whole tube the flow stalls... ." Actually Eckart's solution is not valid in the limit as the acoustic beamwidth approaches the tube diameter. The breakdown in the solution is the result of Eckart's deletion of oscillatory vorticity generated by the acoustic beam. Hence, as the beam is widened to within a Stokes boundary layer thickness of the wall, Eckart arrives at zero value for gradient of the time

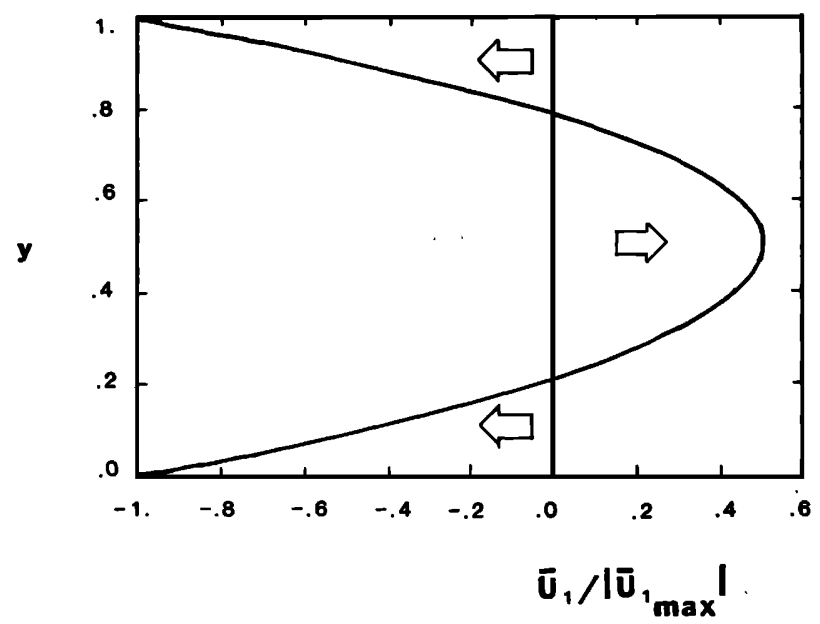

FIG. 3. Streaming motion in a duct having uniform height $h=1$ with $R_{f}=0$ and $\gamma^{\prime}=k \cdot \bar{u}_{1} /\left|\bar{u}_{1_{\max }}\right|$ is plotted versus $y$. 
averaged Reynolds stress. Therefore there is no source to drive the streaming motion in the enclosed fluid.

The unsteady pressure and velocities contain first and second harmonic components. Harmonic components are determined by solving the homogeneous equation and the second harmonic components are determined by an inhomogeneous set of equations. Since the second harmonic components are $O(1 / S)$, the result will not be presented here. Following the solution process outlined in Sec. III the harmonic solutions are

$$
\begin{aligned}
u_{1}= & \operatorname{Re}\left[-i p_{1}^{(1)}\left(1+e^{-A y}-e^{-A \tilde{y}}\right) e^{-i t}\right] \\
& +O\left(e^{-A h}\right)+O\left(e^{-i 2 t} / S\right) \\
v_{1}= & \operatorname{Re}\left\langle\left( i w_{1} y+\left[i p_{1 x x}^{(1)}\left(e^{-A y}-e^{-A \tilde{y}}-1\right) / A\right]\right.\right. \\
& \left.\left.+p_{1 x}^{(1)} h^{\prime} e^{-A \tilde{y}}\right\} e^{-i t}\right\rangle+O\left(e^{-\alpha h}\right)+O\left(e^{-i 2 t} / S\right),
\end{aligned}
$$

where

$$
\begin{aligned}
& \left(p_{1}^{(1)} h^{\prime}\right)^{\prime}+k^{2} p_{1}^{(1)}-p_{1}^{(1) \cdots} / A \\
& =O\left(e^{-i 2 t} / S\right)+O\left(e^{-A h}\right), \\
& A=\alpha(1-i) \text {, } \\
& p_{1}=\operatorname{Re}\left[p_{1}^{(1)}(x, k) e^{-i t}\right]+O\left(e^{-i 2 t} / S\right)+O\left(e^{-\alpha h}\right) \text {, } \\
& w_{1}=p_{1}^{\prime \prime(1)}+k^{2} p_{1}^{(1)} \text {, } \\
& \alpha=(R / 2)^{1 / 2} \text {, }
\end{aligned}
$$

and

$$
\tilde{y}=h-y .
$$

\section{SECOND-ORDER COEFFICIENT}

Equating like terms in $\epsilon^{2}$ we can generate the equations that govern the values of the second order coefficients. The unsteady equations are

$$
\begin{aligned}
& \dot{u}_{2}+(1 / S)\left[\left(u_{0} u_{1}\right)_{x}+v_{0} u_{1_{y}}+v_{1} u_{0_{y}}-\left(\overline{u_{0} u_{1}}\right)_{x}-\overline{v_{0} u_{1 y}}\right. \\
& \left.\quad-\overline{v_{1} u_{0_{y}}}\right]+\left(1 / S^{2}\right)\left[\left(\bar{u}_{1} u_{0}\right)_{x}+\bar{v}_{1} u_{0_{y}}+\bar{u}_{1_{y}} v_{0}\right]+p_{2_{x}} \\
& \quad=(1 / R)\left(u_{2_{y y}}+u_{0_{x x}}\right) \\
& \dot{v}_{0}+p_{2_{y}}=(1 / R) v_{0_{y y}}
\end{aligned}
$$

and

$$
u_{2_{x}}+v_{2_{y}}=k^{2} \dot{p}_{2} .
$$

The steady equations of motion are

$$
(1 / S)\left[\left(\overline{u_{0} u_{1}}\right)_{x}+\overline{v_{1} u_{0_{y}}}+\overline{v_{0} y_{1_{y}}}\right]+\bar{p}_{2_{x}}=(1 / R S) \bar{u}_{2 y y} \text {. }
$$

$\bar{p}_{2_{y}}=0$,

$\bar{u}_{2_{x}}+\bar{v}_{2_{y}}=0$.

From our development in Sec. IV, we saw that $u_{1}$ and $v_{1}$ contained harmonic and second harmonic terms. The only source terms in Eqs. (54) and (55) that will remain after the time average is taken are the products of the harmonic components. Therefore, as far as second-order streaming velocities are concerned, deleting the evaluation of the second harmonic terms in $u_{1}$ and $v_{1}$ in Sec. IV is justified. Replacing $u_{1}$ and $v_{1}$ with the harmonic components $u_{1}^{(1)}$ and $v_{1}^{(1)}$ in Eqs.
(54) and (55) yield

$$
\begin{aligned}
& (1 / S)\left[\left(\overline{u_{0} u_{1}^{(1)}}\right)_{x}+\overline{v_{1}^{(1)} u_{0_{y}}}+\overline{v_{0} u_{y_{y}}^{(1)}}\right]+p_{2_{x}} \\
& \quad=(1 / R S) \bar{u}_{2_{y y}}, \\
& \bar{u}_{2_{x}}+\bar{v}_{2_{y}}=0 .
\end{aligned}
$$

The solution for $\bar{u}_{2}$ and $\bar{v}_{2}$ takes the same form as $\bar{u}_{1}$ and $\bar{v}_{1}$. After following the same solution process as in Sec. IV the streaming velocities are

$$
\begin{aligned}
\bar{u}_{2} \simeq & \left(\frac{R S}{2} \bar{p}_{2}^{\prime}+\frac{R}{2} B_{1}\right)\left(y^{2}-h y\right)+B_{1}\left[\phi_{1_{y}}-\left(\frac{3}{2}-\frac{2}{h \alpha}\right)\right] \\
& -B_{2}\left[\phi_{2_{y}}-\left(-\frac{1}{2}+\frac{2}{h \alpha}\right)\right]+B_{3} \phi_{3_{y}}+O\left(e^{-A h}\right), \\
\bar{v}_{2} \simeq & -\left[\left(\frac{R S}{2} \bar{p}_{2}^{\prime}+\frac{R}{2} B_{2}\right)\left(\frac{y^{3}}{3}-\frac{h y^{2}}{2}\right)\right]_{x} \\
& -\left\{B_{1}\left[\phi_{1}-\left(\frac{3}{2}-\frac{2}{h \alpha}\right) y\right]\right]_{x} \\
+ & \left\{B_{2}\left[\phi_{2}-\left(-\frac{1}{2}+\frac{2}{\alpha h}\right) y\right]_{x}\right\} \\
& -\left[B_{3}\left(\phi_{3}-\frac{h^{\prime}}{h \alpha} \frac{3}{2}\right)\right]-B_{3} \frac{k^{2}}{2 \alpha}\left(\phi_{4}-1\right)+O\left(e^{-\alpha h}\right),
\end{aligned}
$$

where

$$
\begin{aligned}
& B_{1}= \operatorname{Re}\left(p_{0}^{* \prime} p_{1}^{(1) \prime \prime}+p_{1}^{*(1) \prime} p_{0}^{\prime \prime}\right) / 2 \\
& B_{2}=\operatorname{Im}\left(p_{0}^{* \prime} p_{1}^{(1) \prime \prime}+p_{1}^{*(1) \prime} p_{0}^{\prime \prime}\right) / 2, \\
& B_{3}=\operatorname{Re}\left(p_{0}^{* \prime(1)} p_{0}^{\prime}\right), \\
&\left(\frac{R S}{2} \bar{p}_{2}^{\prime}+\frac{R}{2} B_{1}\right)=-B_{1}\left(\frac{9}{h^{2}}-\frac{21}{\alpha h^{2}}\right) \\
&-B_{2}\left(\frac{36}{h^{4} \alpha^{2}}+\frac{3}{h^{2}}-\frac{21}{\alpha h^{3}}\right) \\
&+B_{3}\left(\frac{h^{\prime}}{h^{4} \alpha} 18\right),
\end{aligned}
$$

and $\phi_{1}, \phi_{2}, \phi_{3}, \phi_{4}$ and their derivatives in $y$ are given in Eqs. (37) and (38).

For large Reynold numbers, the second-order streaming velocities outside the Stokes layer are

$u_{2} \sim-\left(B_{1} \frac{3}{2}+\frac{B_{2}}{2}\right)\left[1+6\left(\frac{y^{2}}{h^{2}}-\frac{y}{h}\right)\right]+O\left(\frac{1}{\alpha}\right)$,

$\bar{v}_{2} \simeq\left\{\left(B_{1} \frac{3}{2}+\frac{B_{2}}{2}\right)\left[y+5\left(\frac{y^{3}}{3 h^{2}}-\frac{y^{2}}{2 h}\right)\right]\right\}_{x}+O\left(\frac{1}{\alpha}\right)$,

and

$$
\bar{\psi}_{2}=-\left(B_{1} \frac{3}{2}+\frac{B_{2}}{2}\right)\left[y+6\left(\frac{y^{3}}{3 h^{2}}-\frac{y^{2}}{2 h}\right)\right] .
$$

The result is functionally identical to that obtained in Sec. IV. However, the amplitudes differ. The values for pressure at this order depend on the impedance boundary conditions downstream; i.e., the terminal impedance. If the terminal impedance is independent of $\epsilon$ the acoustic streaming at this order is zero.

The approximate solutions for the harmonic compo- 
nents of the second order particle velocities and pressure are

$$
\begin{aligned}
u_{2}= & \operatorname{Re}\left[-i F^{\prime}\left(1-e^{-A y}-e^{-A \bar{y}}\right)+\frac{1}{2} i w_{0}^{\prime}\left(y^{2}-h^{2} e^{-A \bar{y}}\right)\right. \\
& \left.+i C \bar{y} e^{-A \tilde{y}}\right] e^{-i t}+O(1 / \alpha)+O(1 / S), \\
v_{2}= & \operatorname{Re}\left[\left(i w_{2} y+F^{\prime} h^{\prime} e^{-A \tilde{y}}+i \frac{F^{\prime \prime}}{A}\left(e^{-A y}-e^{-A \bar{y}}-1\right)\right.\right. \\
& -i\left[\left(\frac{1}{6}\right)\left(w_{0}^{\prime \prime}+k^{2} w_{0}\right)\left(y^{3}\right)+\left(\frac{1}{6}\right)\left(h^{3}\right)^{\prime} w_{0} e^{-A \bar{y}}\right] \\
& \left.\left.+i\left(C h^{\prime}-C^{\prime} / A\right) \tilde{y} e^{-A \tilde{y}}\right) e^{-i t}\right]+O(1 / \alpha)+O(1 / S)
\end{aligned}
$$

and

$$
p_{2}=F+w_{0} y^{2} / 2+p_{0}^{\prime \prime} y / A+O(1 / \alpha),
$$

where

$$
\begin{aligned}
& w_{0}=p_{0}^{\prime \prime}+k^{2} p_{0}, \\
& w_{2}=F^{\prime \prime}+k^{2} F, \\
& \tilde{y}=h-y, \\
& C=\left\{w_{0} h-h^{\prime}\left[\left(A w_{0} h\right)-p_{0}^{\prime \prime}\right]\right\} / 2,
\end{aligned}
$$

and

$$
\begin{aligned}
& \left\{F^{\prime}[h-(2 / A)]\right\}^{\prime}+h k^{2} F \\
& =\left[\left(w_{0}^{\prime} h^{3}\right) / 6\right]^{\prime}+\left(h^{3} k^{2}\right) w_{0} / 6 \\
& \quad+O(1 / \alpha)+O(1 / S) .
\end{aligned}
$$

Contributions to the oscillatory velocity and pressure resulting from the convective acceleration terms

$$
\begin{gathered}
1 / S\left[\left(u_{0} u_{1}\right)_{x}+v_{0} u_{1_{y}}+v_{1} u_{0_{y}}-\left(\overline{u_{0} u_{1}}\right)_{x}-\overline{v_{0} u_{1_{y}}}\right. \\
\left.\quad-\overline{v_{0} u_{1_{y}}}-\overline{v_{1} u_{0_{y}}}\right],
\end{gathered}
$$

and

$$
1 / S^{2}\left[\left(\bar{u}_{1} u_{0}\right)_{x}+\bar{v}_{1} u_{0_{y}}+\bar{u}_{1_{y}} v_{0}\right]
$$

were neglected in evaluating $u_{2}, v_{2}$, and $p_{2}$. Therefore, our error in the evaluation of $u_{2}, v_{2}$, and $p_{2}$ is $O(1 / \alpha)+O(1 / S)$.

\section{THIRD-ORDER COEFFICIENT}

Retaining only the $\epsilon^{3}$ terms in Eqs. (20)-(22) yields the third-order streaming equations. The steady equations are

$$
\begin{aligned}
& (1 / S)\left(\overline{u_{1} u_{1_{x}}}+\overline{v_{1} u_{1_{y}}}\right)+(1 / S)\left[\left(\overline{u_{2} u_{0_{y}}}\right)+\overline{v_{2} u_{0_{y}}}\right. \\
& \left.\quad+\overline{v_{0} u_{2_{y}}}\right]+\left(1 / S^{3}\right)\left(\bar{u}_{1} \bar{u}_{1_{x}}+\bar{v}_{1} \bar{u}_{1_{y}}\right)+\bar{p}_{3_{x}} \\
& \quad=(1 / R S)\left(\bar{u}_{3_{y y}}+\bar{u}_{1_{x x}}\right) \\
& (1 / S)\left(\overline{u_{0} v_{0_{x}}}+\overline{v_{0} v_{0_{y}}}\right)+\bar{p}_{3_{y}}=(1 / R S) \bar{v}_{1_{y y}}, \\
& \bar{u}_{3_{x}}+\bar{v}_{3_{y}}=0 .
\end{aligned}
$$

Now let us concentrate our effort on the streaming velocities driven by

$$
\left(\bar{u}_{1} \bar{u}_{1_{x}}+\bar{v}_{1} \bar{u}_{1_{y}}\right) \text {. }
$$

It is this term that determines the maximum amplitude for which our perturbation solution is valid. Deleting all time average terms from Eqs. $(63)-(65)$ yield

$$
\begin{aligned}
& \frac{1}{S^{3}}\left(\bar{u}_{1} \bar{u}_{1_{x}}+\bar{v}_{1} \bar{u}_{1_{y}}\right)+\bar{p}_{3_{x}}=\frac{1}{R S}\left(\bar{u}_{3_{y y}}+\bar{u}_{1_{x x}}\right), \\
& \bar{p}_{3_{y}}=(1 / R S) \bar{v}_{1_{y y}}, \\
& \bar{u}_{3_{x}}+\bar{v}_{3_{y}}=0 .
\end{aligned}
$$

The approximate streaming velocity in the horizontal direction resulting from the term $1 / S^{2}\left(\bar{u}_{1} \bar{u}_{1_{x}}+\bar{v}_{1} \bar{u}_{1_{y}}\right)$ is

$$
\begin{aligned}
\bar{u}_{3} \simeq & \frac{R}{S^{2}}\left(D D^{\prime}-2 D^{2} h^{\prime}\right)\left[y^{6}\left(\frac{2}{5 h^{4}}\right)-y^{5}\left(\frac{6}{5 h^{3}}\right)\right]+\frac{R}{S^{2}} D D^{\prime}\left(y^{4} \frac{3}{2 h^{2}}-\frac{y^{3}}{h}+y^{2} \frac{1}{2}-\frac{1}{5} y h\right) \\
& -\frac{R}{S^{2}} D^{2} h^{\prime}\left[y^{4}\left(\frac{1}{h^{3}} \frac{3}{2}+\frac{1}{h^{2}}\right)-y^{3} \frac{1}{h^{2}}-\left(\frac{-3}{5} h+\frac{1}{2}\right) y\right] \\
& +f_{1}\left[6 D\left(\frac{y^{3}}{h^{2}}-\frac{y^{2}}{h}+D y\right)\right]_{x}-h^{\prime} f_{2} 6 D\left(\frac{y^{2}}{h}-\frac{y}{h}+D\right)+O\left(\frac{1}{\alpha}\right),
\end{aligned}
$$

where

$$
\begin{aligned}
& D=-A_{1}\left(\frac{3}{2}\right)-A_{2}\left(\frac{1}{2}\right), \\
& f_{1}=A_{3}\left[-h^{\prime} e^{-\alpha y} \sin \alpha y+\left(h^{\prime} y / h\right)\left(e^{-\alpha y} \sin \alpha y+e^{-\alpha y} \sin \alpha y\right)\right], \\
& f_{2}=f_{1}\left(h^{\prime} / h\right) y e^{-\alpha y} \sin \alpha y,
\end{aligned}
$$

and $R / S^{2}=R_{s}$ is the streaming Reynolds number. The streaming motion driven by the convective acceleration term

$$
1 / S\left(\overline{u_{1} u_{1_{x}}}+\overline{v_{1} u_{1_{y}}}\right)
$$

is the same as the result obtained in Sec. IV. The solution for the steady motion can be obtained by simply replacing $p_{0}$ with $p_{1}$ in Eqs. (42) and (43). The deletion of the second harmonic component of $u_{1}, v_{1}$, and $p_{1}$ results in an error of $O\left(\epsilon^{3} / S^{2}\right)$ in the total streaming solution. It can be shown that the solution for the streaming motion driven by the convective acceleration terms

$$
1 / S\left(\overline{u_{0} v_{0_{x}}}+\overline{v_{0} v_{0_{y}}}\right)
$$

and

$$
1 / S\left[\left(\overline{u_{2} u_{0}}\right)_{x}+\overline{v_{2} u_{0_{y}}}+\overline{v_{0} u_{2_{y}}}\right]
$$

yield results similar to those obtained in Sec. V. We need only replace $p_{1}$ with $F$ in Eqs. (58) and (59). The error in the streaming motion using the aforementioned replacement is $O\left(\epsilon^{3} / S^{2}\right)$. The complete third-order coefficient for stream function outside 
the boundary layer is

$$
\begin{aligned}
\bar{\psi}_{3}= & \frac{R}{S^{2}}\left(D D^{\prime}-2 D^{2} h^{\prime}\right)\left[\frac{y^{7}}{h^{4} 15}-\frac{y^{6}}{h^{3} 5}-\frac{4}{5}\left(\frac{y^{3}}{3}-\frac{h y^{2}}{2}\right)\right]+\frac{R}{S^{2}} D D^{\prime}\left[\frac{3}{10} \frac{y^{5}}{h^{2}}-\frac{y^{4}}{4 h}+y^{3}\left(\frac{6}{15}\right)-h y^{2}\left(\frac{9}{10}\right)\right] \\
& -\frac{R}{S^{2}} D^{2} h^{\prime}\left[y^{5}\left(\frac{3}{h^{3} 10}+\frac{1}{h^{2} 5}\right)-\frac{y^{4}}{4 h^{2}}+y^{3}-\left(\frac{-3 h}{10}+\frac{1}{4}\right) y^{2}+\left(\frac{-6}{h 5}+3\right)\left(\frac{y^{3}}{3}-\frac{h y^{2}}{2}\right)+y^{2}\left(\frac{13}{20}\right)\right] \\
& -\left(E_{1} \frac{3}{2}+E_{2} \frac{1}{2}\right)\left[y+6\left(\frac{y^{3}}{3 h^{2}}-\frac{y^{2}}{2 h}\right)\right]+O\left(\frac{1}{S^{2}}\right) \\
& -\left(G_{1} \frac{3}{2}+G_{2} \frac{1}{2}\right)\left[y+6\left(\frac{y^{3}}{3 h^{2}}-\frac{y^{2}}{2 h}\right)\right]+O\left(\frac{1}{S^{2}}\right)+O\left(\frac{1}{\alpha}\right),
\end{aligned}
$$

where

$$
\begin{aligned}
& D=-A_{1}(3 / 2)-A_{2}(1 / 2), \\
& E_{1}=\operatorname{Re}\left(p_{1_{x}}^{*(1)} p_{1_{x x}}^{(1)}\right) / 2, \\
& E_{2}=\operatorname{Im}\left(p_{1_{x}}^{*(1)} p_{1_{x x}}^{(1)}\right) / 2, \\
& G_{1}=\operatorname{Re}\left(p_{0}^{* \prime} F^{\prime \prime}+F^{* \prime} p_{0}^{\prime \prime}\right) / 2,
\end{aligned}
$$

and

$$
G_{2}=\operatorname{Im}\left(p_{0}^{* \prime} F^{\prime \prime}+F^{* \prime} p_{0}^{\prime \prime}\right) / 2 .
$$

We can now determine the limiting amplitude for our perturbation solution. If the terminal impedance is independent of $\epsilon$, the perturbation solution is valid until $\epsilon R / S^{2}$ becomes of $O(1)$. This means that our limiting amplitude is dependent on terminal boundary conditions and the acoustic wavelength. Therefore, in a waveguide the maximum velocity amplitude for which the perturbation solution is valid is at least a multiplicative factor of $1 / \sqrt{\epsilon}$ greater than that reported in the literature.

\section{REGION OF VALIDITY OF THE PERTURBATION SOLUTION}

The region of validity of our analysis can be parametrized by "critical values" of the oscillatory Reynolds number $R$, Strouhal number $S$, and the wavelength parameter $\epsilon$. In this section we will present a graphical representation of this region. We will show how the critical value of the streaming Reynolds number relates to all three of the aforementioned parameters.

Recall that in solving for the streaming velocities, we assumed that $R$ and $S$ had large fixed values. By holding these parameters fixed we were able to express the streaming motion terms of a regular perturbation sequence in $\epsilon$. For example the horizontal streaming velocity was expressed as

$$
E_{3} \bar{u}=\epsilon \bar{u}_{1}+\epsilon^{2} \bar{u}_{2}+\epsilon^{3} \bar{u}_{3}+O\left(\epsilon^{4}\right),
$$

where

$$
\begin{aligned}
& \bar{u}_{1}=O(1), \\
& \bar{u}_{2}=O(1), \\
& \bar{u}_{3}=O\left(R / S^{2}\right)+O(1)=O\left(R_{s}\right)+O(1),
\end{aligned}
$$

and $\boldsymbol{R}_{s}$ is the streaming Reynolds number which is equal to $U_{0}^{2} / \omega v$.

The zeroth-order velocity $\bar{u}_{0}$, is equal to zero. This situation resulted from the assumption that the guide enclosed no sources of steady momenta other than those induced by the acoustic wave.
In Sec. VI it was shown that part of third-order coefficient $\bar{u}_{3}$ was proportional to $R / S^{2}$ which is the streaming Reynolds number $\boldsymbol{R}_{s}$. A nonuniformity in our asymptotic expansion occurs when $\bar{u}_{3}=O\left(\epsilon \bar{u}_{2}\right)$. Expressing this condition of $\boldsymbol{R}_{s}$ yields

$$
R_{s}=O(1 / \epsilon),
$$

where

$$
R_{s}=R / S^{2}=U_{0}^{2} / \omega v .
$$

Hence, there is a relationship between $R, S$, and $\epsilon$ which bounds the present theory. When $R_{s}$ becomes $O(1 / \epsilon)$, the steady and unsteady equations become coupled and nonlinear. For $R_{s}$ less than $O(1 / \epsilon)$, the streaming solution presented is valid.

At this point it is important to note that the bound on $R_{s}$ given in Eq. (70) differs from the bounds given by Wang ${ }^{4}$ and Secomb. ${ }^{5}$

Wang assumed that his viscous incompressible solution for acoustic streaming was in the regime where $V_{0}=O\left(U_{0}\right)$, and $\omega / c=0$ reflected in the same character as the compressible flow solution. Hence, he arrived at the conclusion that the critical value of the streaming Reynolds number occurs when $R_{s}=O(1)$. This process is equivalent to setting $k=0$ in our equations and letting $\epsilon$ go to 1 in our analysis.

Secomb analyzed the problem of acoustic streaming in a viscous incompressible fluid enclosed in a channel. $\mathrm{He}$ showed that in the long wavelength limit that his acoustic streaming solution was valid to $R_{s}=O(\infty)$. However, in his development he implicitly assumed that $L_{0} \omega / c=O\left(H_{0} / L_{0}\right)$, where $H_{0}$ is the height of the channel and $L_{0}$ is the characteristic wavelength. Therefore, in taking the incompressible limit, $1 / c \rightarrow 0$, he in effect forced $H_{0} / L_{0}$ to go to zero at the same rate. From our analysis we can see what effect the coupling of these two parameters, $L_{0} \omega / c$ which equals $k$, and $H_{0} / L_{0}$, which equals $\epsilon$, has on the tabulation of the critical streaming Reynolds number. Letting $k=0$ and taking the limit of Eq. (70) as $\epsilon$ goes to zero yields

$$
R_{s}=\lim _{\epsilon \rightarrow 0} O(1 / \epsilon)=\infty,
$$

which is Secomb's result.

The region of validity of our result can best be depicted graphically. In Fig. 4 we have plotted the lines where the Strouhal number $S$, the streaming Reynolds number $R_{s}$, and $\epsilon$ are $O(1)$. These lines are plotted as a function of two nondimensional variables $R / S$ and $R$. To the right of the point 


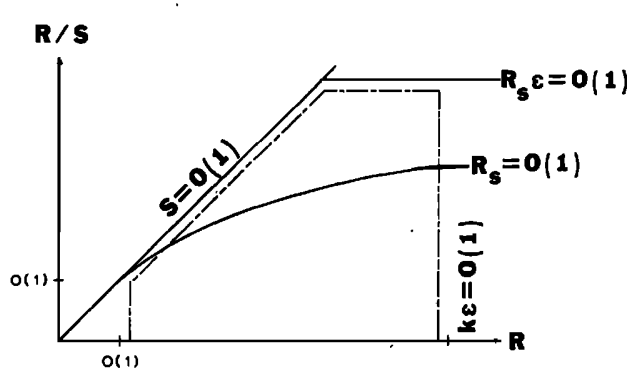

FIG. 4. The region of validity of the perturbation solution.

where $R=O(1)$ defines oscillatory Stokes flow while the region to the left defines oscillatory Poiseuille flow. Above the line $S=O(1)$, the convective terms cannot be treated as second-order quantities. The upper bound oscillatory amplitude is delineated by the line $R_{s}=O(1 / \epsilon)$. The constant $R_{s} \epsilon$ line is parallel to the $R$ axis because $\epsilon$ was assumed to be equal to the reciprocal of the acoustic wavelength times $H_{0}$. Above this line the unsteady and steady flow become nonlinear and coupled. The vertical line where $\epsilon$ is $O(1)$ denotes the critical frequency at which cross modes in the guide become important. The line $R_{s}=O(1)$ delineates the boundary above which classically the steady convective term dominates the analysis.

Our conclusion on the critical value of the streaming Reynolds number can be supported further by the results of a recent experimental investigation which was made by Chow et al. ${ }^{10}$ into the onset of acoustically induced turbulence.

Chow et al. performed experiments to determine the oscillatory amplitude at which turbulent bursting occurred near the wall of a uniform cylindrical tube. The rigid 1-in.diam tube was excited harmonically with a plane traveling acoustic wave. Experiments were carried out in the frequency range between 500 and $2200 \mathrm{~Hz}$, and the amplitude varied from 150 to $168 \mathrm{~dB}$ re: $20 \mu \mathrm{Pa}$. In this range the typical oscillatory Reynolds number is about $10^{5}$ and the Strouhal number is about $10^{3}$ when based on tube diameter. They found that intermittent turbulent bursting in the oscillatory particle velocity, as measured by a hot film probe, occurred at a sound pressure level of $158 \mathrm{~dB}$. They observed that this critical pressure amplitude remained constant with variations in frequency. This sound pressure level corresponded to a peak horizontal particle velocity $U_{0}$, of $5.52 \mathrm{~m} / \mathrm{s}$. Let

$$
\epsilon=d / \lambda \text {, }
$$

where $d=1$ in., the diameter of the tube and $\lambda=$ the acoustic wavelength.

The amplitude parameter $\left(R_{s} \epsilon\right)^{1 / 2}$, can be computed as

$$
\left(\epsilon R_{s}\right)^{1 / 2}=U_{0} \sqrt{d} /(2 \pi v c)^{1 / 2}=4.7,
$$

where

$$
\begin{aligned}
& v=1.59 \times 10^{-5} \mathrm{~m}^{2} / \mathrm{s}, \\
& c=345 \mathrm{~m} / \mathrm{s}, \\
& d=1 \text { in. }=(2.54 \mathrm{~cm}) /(100 \mathrm{~cm} / \mathrm{m}),
\end{aligned}
$$

and

$$
U_{0}=5.52 \mathrm{~m} / \mathrm{s}
$$

for the frequency range between 500 and $2200 \mathrm{~Hz}$. Therefore, our observation that the value of $\epsilon R_{s}=O(1)$ results in the coupling of steady and unsteady equations appears to be a valid one.

\section{ACKNOWLEDGMENTS}

The author is indebted to Professor Patrick Leehey and Professor Mack A. Breazeale for valuable suggestions on earlier versions of this manuscript.

\footnotetext{
'Lord Rayleigh, Philos. Trans. A175, 1 (1883).

${ }^{2}$ R. R. Haselton and P. W. Schuer, Science 208, 69 (1980).

${ }^{3}$ C. G. Caro, J. M. Fitzgerald, and R. C. Schroter, Nature 223, 1159 (1969).

${ }^{4} \mathrm{C}$. Wang, J. Fluid Mech. 32, 55 (1968).

${ }^{5}$ T. W. Secomb, J. Fluid Mech. 88, 273 (1972).

${ }^{6}$ H. Schlichting, Phys. Z. 23, 327 (1932).

${ }^{7}$ N. Riley, J. Inst. Math. Appl. 3, 419 (1967).

${ }^{8}$ J. T. Stuart, J. Fluid Mech. 24, 673 (1966).

${ }^{9}$ C. Eckart, Phys. Rev. 73, 68 (1948).

${ }^{10}$ K. H. Chou, P. S. Lee, and D. T. Shaw, J. Acoust. Soc. Am. 68, 1780 (1980).
} 